\title{
Im Gespräch bleiben - Dysarthrie bei Morbus Parkinson
} Patienteninformation

Die Parkinson-Erkrankung gehört zu den häufigsten degenerativen Erkrankungen, in deren Verlauf eine Dysarthrie, eine Sprechstörung, auftritt. Charakteristisch ist die leiser werdende, monotone Sprechweise, verbunden mit ungenauen, kleinen und daher bisweilen schnellen Sprechbewegungen. Alle am Sprechablauf beteiligten Muskelgruppen - die der Atmung, der Stimmgebung und der Lautbildung - werden im Bewegungsfluss steifer und damit kleiner.

Folgen der Dysarthrie
> sozialer Rückzug
- verringerter kommunikativer Aus-
tausch
- häufige Missverständnisse
- kommunikative und emotionale
Verarmung
- zunehmende Depression

\section{Verständlichkeit - eine Sache der Lautstärke \\ $\nabla$}

Ramig und Mitarbeiter beobachteten, dass die Verständlichkeit der ParkinsonPatienten in erster Linie durch die geringe Lautstärke beeinträchtigt ist. Die leiser werdende Stimme ist in der Regel das 1. Symptom der Dysarthrie. Der langsam einsetzende Prozess lässt oft den Beginn der Dysarthrie unbemerkt, und es erfolgt eine Gewöhnung an die leise Stimme. Zusätzlich verlieren die Patienten die Fähigkeit, im Augenblick des Sprechens die Lautstärke oder das Sprechtempo zu beeinflussen.

Medikamente und tiefe Hirnstimulation Die medikamentöse Behandlung und die tiefe Hirnstimulation verbessern das Sprechen nur begrenzt: Die Verringerung des Tremors führt in der Anfangsphase zu einer stabileren Stimme. Wird im Verlauf die Dosis oder die Stimulation erhöht, was oft zu besserer Beweglichkeit führt, kann sich dies negativ auf das Sprechen auswir- ken: Unter der Stimulation verweist eine stärker verwaschene Sprechweise oder eine gepresst heisere, angestrengt klingende Stimme auf eine ungünstige Einstellung.

\section{Wirksame logopädische Behand- lungsverfahren \\ $\nabla$}

„Sei laut!“ Das Lee-Silverman-VoiceTreatment (LSVT) steigert systematisch die Lautstärke des Patienten hin zu einer normal lauten, kraftvollen Stimme. Aufgrund täglicher Übungsaufgaben und hierarchisch aufgebauten Anwendungsschritten für den Alltag kann die wieder gefundene, kraftvolle Stimme im Gespräch eingesetzt werden. Unter der Devise "Sei laut“ lernt der Patient, sich im Alltag selbst den Impuls zu geben, laut zu sprechen. 4 Wochen mit 4 Therapien pro Woche reichen aus, um eigenständig weiter zu arbeiten und im Alltag hinlänglich laut zu sprechen. Selbstverständlich hängt dies vom Krankheitsstadium ab, aber ein Mindestmaß an Verbesserung gelingt in jedem Stadium. Ein früher Therapiebeginn und Auffrischungen bei nachlassender Stimmkraft verbessern die Chancen für erfolgreiche Kommunikation (http:// www.lsvtglobal.com).

Hilfsmittel Stimmverstärker funktionieren wie ein Mikrofon mit Lautsprecher und ermöglichen bei gut erhaltener Lautbildung eine erfolgreiche Kommunikati-

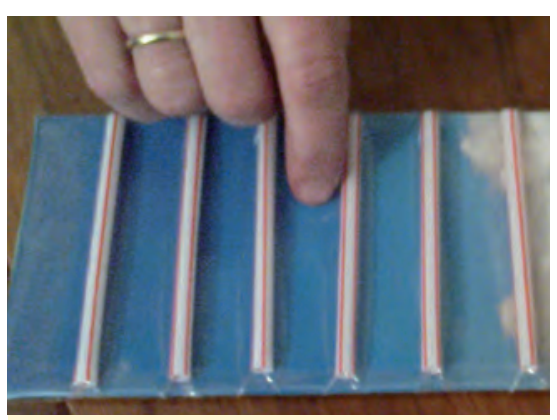

Abb. 1 Tastbrett. on. Sie verlangen jedoch technische Kompetenz von Patienten und Umfeld.

Einfach und erfolgreich ist das sogenannte Tastbrett von Nancy Helm ( $\bullet$ Abb. 1). Um das Sprechtempo zu verringern, wird bei jedem Wort zwischen die Markierung getippt (wie auf einer Leiter). Bei systematischer Übung auch mit den Angehörigen kann die Verständlichkeit rasch verbessert werden. Allerdings muss das „Tastbrett" stets verwendet werden.

Täglich üben hilft Jedes Verfahren erfordert einen Lernprozess, der für alle Parkinson-Patienten aufgrund des Dopaminmangels schwierig ist. Deswegen erfordert jedes Verfahren eine intensive, d.h. tägliche, Übungsphase über einen relativ kurzen Zeitraum (4 Wochen). Die eigenständige Übung erfordert anfangs tägliche Rückkoppelung, um das Lernen von Fehlern zu vermeiden. Nur eine gesicherte Bewertung der Lautstärke oder des Sprechtempos kann zu einer erfolgreichen Kommunikation führen.

\section{Fazit \\ - Die Dysarthrie der Parkinsonkrank- heit erschwert erheblich die Kommu- nikation. \\ - Ein früher Behandlungsbeginn ist dringend zu empfehlen. \\ - Als Methoden eignet sich besonders das Lee-Silverman-Voice-Treatment (LSVT) oder das Tastbrett. \\ - Lernprozesse erfordern eine hohe Therapiefrequenz.}

Adelheid Nebel, Kiel

überreicht durch Thieme und 